\title{
People Portraits and Cartoon Algorithm Study
}

\author{
Weihua Gai \& Dakun Zhang \\ College of Computer Technology and Automation \\ Tianjin Polytechnic University \\ Tianjin 300160, China \\ E-mail: gaiweihua.1-3@163.com
}

\begin{abstract}
This paper is to use the positive face to automatically generate a photo realist style, or has the effect of exaggerated cartoons. It includes two aspects: First, face the portrait photos automatically into production; Second, face deformed to generate cartoon effect. AAM model is a statistical model, can be well applied to the facial characteristics of location. In this paper, by improving the traditional AAM ways to overcome its lack of color information processing, design a more effective figure portraits Generation System. Then, take the initiative to shape a model feature extraction and characteristics of the method of combining the human face of the main features of automatic deformation, the cartoon characters generated portrait.
\end{abstract}

Keywords: Portrait photos, AAM model, Deformation, Cartoon

\section{Introduction}

In recent years, digital media technology is gradually integrated into daily life, greatly affecting people's lives and work methods. Use of computer-generated cartoon figure portraits in many areas with a wide range of applications such as video conferencing, online games, mobile digital entertainment.

In a variety of applications in the area of a typical portrait of the characters is generated automatically, but mainly in the form of comic books, including painting lines and Oil cartoons. Painting lines in the area, MIT's Brennan was first proposed a comic exaggeration of the interactive generation system. Murakami(1997, p.1), who proposed a template-based production lines painting methods and the development of the PICASSO system, in this method, the comic exaggeration of the extent to adjust. Rein-Lien Hsu(2003, p.2) proposed an interactive model of the Snake adults face the next life painting lines (without exaggeration effect), and it applied to the face in the match. Li, who made an automatic Face painting lines Generation System, the main use of symmetry operator, rectangular outline of filters and features to Face detection and location of the feature points. And various other methods, Microsoft's Chen Hong(2004, p.3), who proposed a case-based method of generating the lines painted. Through the study of the artist's works are photographs Face painting lines to the relationship, when studying the works include exaggerated effect, the formation of the lines are painted with exaggerated effect. Comic lines with the formation of different paintings, Pei-Ying Chiang, who put on an MPEG 4 color portraits of comic exaggeration of the self-generation system. Their systems to have a portrait of comic prototype works, through exaggeration and distortion so as to be processed and the face match, which are the human face of the comics. Through the use of different styles of work as a prototype, could be the result of different styles.

With the previous technology is different, mainly in the improvement of the AAM model based on the design and realization of a figure portraits generation system. That is, not using an interactive model of the Snake did not use symmetry operator, rectangular outline of filters and features to Face detection and location of the feature points way but to use the model based on colour AAM model Face positioning methods access to various features of Facial At the precise location. And the system is a common face for the photo rather than using the prototype has a portrait of comic prototype works to be face cartoons.

\section{System Overview}

This is the main objective is to achieve portraits of the characters automatically generated and exaggeration, to have a cartoon effect, it formed mainly by three steps: The first step is to face the automatic positioning. Here, we used to improve the AAM method. AAM traditional method of its output does not include the nine characteristics of the forehead, improved, people can get the whole face shape. The second step is painting a portrait illustrations. We use 
Unsharp Mask sharpening handling of the color scale computing method, this approach not only take into account mobile device users on the requirements of computing speed, but also to retain the vast majority of people face characteristics, thus more effective in adults face a portrait of Health Illustrations painting. The third step is painting a portrait illustrations deformation generate a certain entertainment humor comics.

\section{Face automatically generated portrait}

People generated portraits of the structure as shown in Figure 1.

Shown in Figure 1 face portraits structure of the generation system. That is to enter a positive face of color photos, and then in accordance with this picture, respectively, to find the appropriate regional and facial hair organs such as eyes, nose and lips, then its final composition of the figures we are portraits system.

In this system, the whole head was divided into regional and facial hair region in two parts. The text of the main difficulties is to determine how accurate and effective regional hair extracted from hair outline and reasonable and in the face of regional coordination of the various feature points. Below will be first introduced hair region and its outline of the extraction method.

\section{1 hair treatment}

As dark hair color has a special nature, that is, the hair pixels $\mathrm{Y}$, the value to meet: $\mathrm{Y} \leq \mathrm{Y} 0, \mathrm{Y} 0$ is a constant. Therefore, we can not satisfied of this nature will be removed pixels, the hair will inevitably fall on the remaining area, from where the hair will be extracted from the region, tracking the region's profile to be the outline of the hair. Concrete steps are as follows:

1) Face Image in the calculation of the various pixels $Y$, values, does not meet the conditions for removal of pixels, the value of the plans are assumed to be G..

2) $G$ for mathematical morphology in the closing operation, the hair of the noise (white hole) filtered by the value of the map.

3) Can be used mathematical morphology of the method, the value of the corrosion plans to conduct two operations. Through corrosion computing, image width of a line of connectivity will be cut off.

4) Tracking the value of the contour map, one for each contour rectangular region ri, with a regional list of $\mathrm{R}=(\mathrm{r} 1$, $\mathrm{r} 2, \ldots, \mathrm{rm}), \mathrm{rm}$ is the number of outline.

5) According to ri determine the contours of regional hair.

6) The outline of the region by the hair line, using seed filling method of filling the hair of the region.

\subsection{Face organ dealing with}

The use of a series of facial images AAM model. In the AAM model, Face the characteristics of a total of 84 points. Active in the shape model (ASM) - On the foundation, T. F. Cootes(1995, p.5\&1998, p.6), who in 1998 proposed the initiative apparent model (AAM). AAM model can be effective use of information and the shape of the object gray change information for the positioning feature points, but the color images feature points Positioning undoubtedly overlook the color information for. Based on the model adopted by the human eye colour location method to determine the human eye rough location and direction, as people face the pattern of distribution of facial features, which can determine the location and who Face direction, which can search for AAM to provide a model Better initial position and size, and can reduce the size of AAM search window, thus reducing the search time AAM. The following was the eye as an example to describe the method of concrete steps:

(1) determine the use of colour model facial image in the eyes of the position;

(2) pupil under the eyes of the connection point and length to determine the initial AAM model size and direction. As long as AAM model known face in the eyes of that special

Levy points of reference to the model zoom and rotate so that they will reach a better initial position;

(3) under the eyes and facial region, identified AAM model search window. The eyes and facial regional relations through more than 20 people from the face image analysis, we will position these images under the eyes of alignment, and then for the average person faces and get the average person in the eyes and face of the geometric face of regional relations.

(4) use AAM model search, facial features, are the exact location.

\section{Deformation exaggerated}

\section{1 deformation}

There are many ways can be used for facial image deformation, and facial animation, more commonly used method for the deformation of the two mesh deformation methods(1998, p.7) and methods based on the characteristics of the 
deformation(1992, p.8). Grid-based technology is deformed image deformation technology in the first one of the ways. Based on the characteristics of the deformation method in the image selected features of the corresponding point line right, the use of the source image in the feature points in the image and objectives of the characteristics of the corresponding point, the characteristics of the region around the pixels are subject to different levels of impact The method can be deformed in high-level control of the process. From the perspective of computing speed in terms of grid computing deformation faster.

The algorithm has been exaggerated deformation often neglected and the length of three Court of facial, which is exaggerated cartoonist for the important factors. The literature [6] is in the face of Grid Group, a large number of feature points to change the coordinates. Feature points are divided into lead, with the correction from the point and three points. Three types of mutual influence between the positioning process is rather complicated. To simplify the process of deformation, this form of the deformation of classification, based on deformation and deformation step-by-step approach to achieve the simplified grid model of purpose. Grid under the automatically generated key feature points, layered automatically exaggerated deformation, and to consider the three Chambers, such as the art of facial exaggeration. Exaggeration deformation is the main form of compression and tension Larger fish eyes squeezed.

Intuitive, the facial features (facial, the three Chambers, the people) of deformation through the stretch or compressed form, and local features (the overall distribution of facial features, eyes, nose, mouth) of the deformation is the use of fish eyes Larger or extrusion of the way. According to an exaggeration of different ways, using two kinds of deformation grid, divided into a Step 2.

\section{2 the first step deformation - facial, the three Chambers, the people of exaggeration}

In order to facilitate the contours of the face, the three Chambers, the people in the tensile and compression, a grid model defined as shown in Figure 2. Grid will be key features of the coordinates of points automatically generated.

Face by calculating the ratio can determine if the facial. Facial can be divided into a long face, wide face and facial standards. If a specific human face aspect ratio in the standard human face aspect ratio difference between the normal range, that is the standard facial is not an exaggeration; Otherwise, the judgement in accordance with aspect ratio for wide or face a long face. For different types of facial, with the proportion of the Third Chamber, the deformation of different ways, according to people in the length and the length of the court to determine whether the proportion of people in the exaggerated length.

For the aspect ratio in the face of the normal standard facial differences within the scope of the face of wide unchanged, according to the length of three Court of exaggeration, that is to change the corresponding grid coordinates of points. Facial for the wide face, the face of the whole squash, narrow vertical. Long face the situation more complicated, if the three meet the standards of the Court, on the face of vertical rectangular uniform tension if the court longer, vertical ladder to face (at the end of a long) stretch if the court longer, The vertical ladder to face (at the end of a long) stretch if the Court in relatively long, the face of the "Shanghai" shaped deformation, that is, on the Court of vertical ladder (at the end of a long) stretch, in the Court of vertical rectangle stretching, under the Court of vertical Trapezoid (at the end of a long) stretch. Divisions based on the proportion of specific tensile Face of the Third Court of proportion.

Three people in the Chamber and the deformation of the grid, as shown in table 1.

Grid coordinates of the points of adjustment for specific standards and who Face the face value of the difference with a random value of the product $b, b$ in the range of three Chambers averaged between face-to a certain degree of effect The randomness.

\subsection{The second step deformation - the exaggerated facial features}

This paper used in the deformation method to enlarge the fish eyes and squeeze. According to the Observer cartoonist works, the effect of fish eyes Larger than in the past used to enlarge the effect of a more linear ideal.

First consider the overall effect of facial features, Meixin points from the calculation of the need to face the focal point for long distance and the proportion of the face, that is, in the Court of length and the length of the people and the proportion of long faces. Chinese aesthetic standards Face the corresponding ratio of 7:10, if a particular human face of this ratio exceeded the standard proportions of the human face of the normal differences, the need for facial features whole fish eyes zoom in or out. And then consider the eyes, nose and mouth size. Have been adopted before the three percentage compared with the standard ratio of the notable features of the judgement, here only exaggerated significant feature.

Step 1. Generation as shown in Figure 5 of the finer grid square.

Step 2. And the need to exaggerate the characteristics of the surrounding grid position deformed fish eyes.

Comic portraits are usually below the narrow neck of this paper, mouth and chin following a specific regional centre of the grid points squeeze deformation. Fish eyes and squeeze algorithm is a specific region of the geometric grid point 
deformation. First default overall impact of facial features, eyes, nose and mouth of their respective rectangular region, the regional deformation rectangular area for the largest circular area, the grid coordinates of points from the Cartesian coordinate system conversion to a coordinate system. After the overall characteristics and local features step-by-step deformation, the result is that the basic drawing comic exaggeration effect.

\section{Conclusion}

Through analysis of exaggerated comic portrait of the painting, made in stages based on the deformation of the comic style portraits of human faces generated algorithms, users can be given in accordance with the face photo, through the key features of the interaction point calibration with automatic generation of the exaggerated effects Face positive portrait comic. This algorithm only user interaction to achieve 14 feature points of calibration and drawing style of choice, easy to operate and generate results have a certain randomness, is simple and more efficient. In a deep understanding of drawing comic books, on the basis of a reasonable use of deformation, deformation stages exaggerated, and achieved good results close to the hand-painted. The next step of work including the use of face recognition research results to the corresponding automatically generated key feature points instead of the user's interaction; achieve more image rendering style to meet the individual needs of users; study how to generate arbitrary attitude and perspective of a portrait of comic books, With photos and further improve the exaggerated side effects.

\section{References}

Beier T and Neely S. Feature-based image metamorphosis[C]//Computer Graphics Proceedings, Annual Conference Series, ACM SIGGRAPH, Negg York. 1992: 35-42.

G J Edwards, C J Taylor and T F Cootes. Interpreting Face Images Using Active Appearance Models[c]. In: Proceedings of the $3^{\text {rd }}$. International Conference on Face\& Ge sture Recognition. Washington, DC, USA: IEEE Computer Society. 1998, 300-305.

Jiang Peiying, Liao Wenhong and Li Caiyan. Automatic caricature generation by analyzing facial features[C]//proceedings of 2004 Asia Co nference on Co mputer Vision, Jeju Island, 2004: 89-94.

M Tominaga, eta1. FaciM caricaturing with motion caricaturing inPICASSO system[C]. In: Proceedings of IEEE/ASME Intema-fional Conference on Advanced Intelligent Mechatronics, NewYork, NY, USA: IEEE, 16-20 June 1997.

Pei-Ying Chla, Wen-Hung Liao and Tsai-Yen Li. AUTOMATICCARICATURE GENERATION BY ANALYZING FACIALF ATURES[C]. In: 2004 Asian Conference on Computer Vision, Jeiu Island, Korea, Jan 27-30,2004.

Rein-Lien Hsu and A K Jain. Generating discriminating cartoonfaces using interacting shakes[J]. IEEE Transactions Oil Pattern Analysisand Machine InteHigence, Nov. 2003, 25(11): 138(8-8).

T Cotes, C Taylor, D Cooper, and J Graham. Active Shape Models-Their Training and Application[J]. In: Computer Vision and ImageUnderstan ding, New York, NY, USA : Elsevier Science Inc.January 1995, 61(1): 38-59.

Wolberg G. Image morphing: a survey[J]. Visual Computer, 1998, 14(8/9): 360-372.

Table 1. Figure 2 deformation of the grid points

\begin{tabular}{|c|c|}
\hline Features & Impact on the grid points \\
\hline In court & 2,3 points to the grid spacing \\
Atrium & 4,5 points to the grid spacing \\
The Chamber & 7,8 points to the grid spacing \\
People & 6,7 points to the grid spacing \\
\hline
\end{tabular}

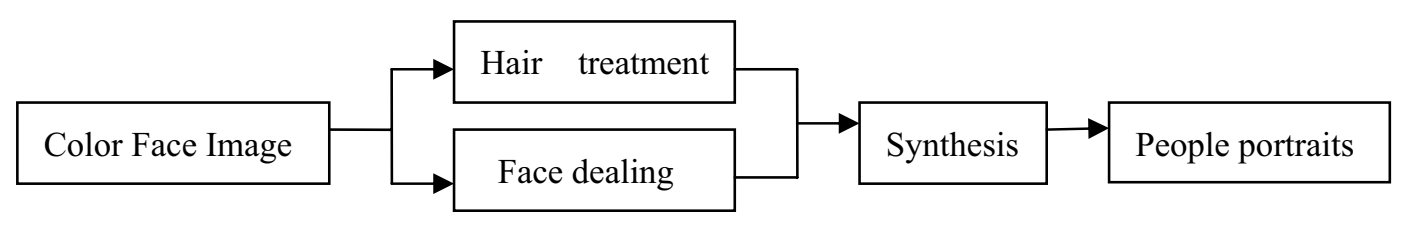

Figure 1. face portraits of the generation system 


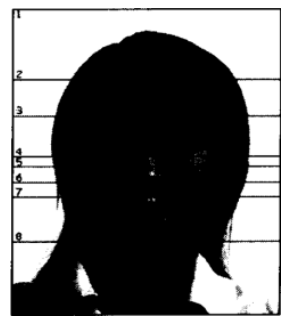

Figure 2. grid model 1

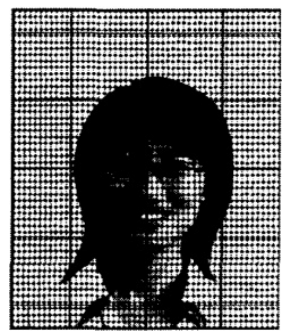

Figure 3. grid model 2 